\title{
Relationship between anthropometric parameters and speed performance: A kinanthropometric research
}

\author{
Metin Can KALAYCI ${ }^{1}$, Ferhat GULEROGLU1', Huseyin EROGLU² \\ ${ }^{1}$ Institute of Health Sciences, Department of Pyhsical Education and Sport, Kahramanmaraş Sütçü Imam University, Kahramanmaraş, Turkey. \\ ${ }^{2}$ Department of Physical Education and Sport, Kahramanmaraş Sütçü Imam University, Kahramanmaraş, Turkey. \\ This study was presented as a report at the International Scientific Conference "Effects of physical activity application to anthropological status with \\ children, youth and adults" University of Belgrade, Faculty of Sport and Physical Education, Republic of Serbia, Belgrade, 11-12 December2014.
}

\begin{abstract}
The purpose in this study; determined of relationship between anthropometric parameters and speed performance. In this purpose; 118 sedentary university students age $21.2 \pm 2.1$ year, body weight $62.2 \pm 10.7 \mathrm{~kg}$ and height $168.2 \pm 8.1 \mathrm{~cm}$ participated in this study voluntarily. $30 \mathrm{~m}$ speed performance with stature (shoulder, chest, trunk, waist, hip, thigh, knee, calf and ankle) and (height, fathoms, upper arm, fore arm, all arms, trunk, thigh, leg and all leg) girths was measured. Statistical analysis was made in SPSS 22.0 packed program for Windows. Data from the measured of arithmetic means, standard deviation, lowest and highest values was found. Relationship between anthropometric parameters and speed performance was tested by Pearson's Correlation. According to Data from the measured; there were significant relationship in between speed values of workgroups and body weight, shoulder girths, chest girths, trunk girths, waist girths ( $<<0.01)$, leg girths $(p<0.05)$ and there were significant relationship in between speed values of workgroup and height, fathoms length, fore arm length, all arm length, trunk length, leg length $(\mathrm{p}<0.01)$, thigh length $(\mathrm{p}<0.05)$. It can be said increases in speed values, if girths and length values increase.
\end{abstract}

Keywords: Anthropometric parameters, speed and sedentary, kinanthropometry.

\section{INTRODUCTION}

Anthropometry is a phrase made of combinations of the words antros and metris (human and measurement). In a general sense, it is a systematic technique classifying objective characteristics of human body by certain measurement methods and principles based on their dimensions and structural characteristics. Today, anthropometry is adopted as a single support in body type and structural characteristic matters (19).Anthropometric techniques may be used for different purposes such as evaluating differences related to the physics between the effects on physical characteristics of training and sports branches (19).

Kinanthropometry can be defined as a scientific field (19) explaining the relationship between human structure and function quantitatively or a discipline (19) researching the relationship between human structure and behavior or examining the effect of human measurement and morphological structure on behavior by considering morphological differences $(17,19)$. It has been considered for many years that a proper body type plays an important role in sportive performance $(16,26)$.

Even in ancient times, studies were performed for examining physical structure of people and according to the results of these, people were classified structurally. Today, the relationship between the physical structure and functions are examined and studies related to the determination of sportive performance are conducted continuously(22).Anthropometric measurements and somatotype has become important for determining skills from the first studies until today(12).Significant developments in all sports branches are a product of the evaluation of fundamental and specific anthropometric and kinesiology characters of athletes(11).Studies have revealed that there are significant differences in physical structures of athletes in different sport branches $(2,8,14,20,24,28,29,32)$.

Measurement of anthropometric characteristics and body composition and revealing their 
relationship with performance is one of the matters that are focused on during the recent years. In many sports branches, it is considered that anthropometric characteristics are one of the most important factors showing whether an athlete could be a competitor at the highest level in that sport $(5,9)$. Making the morphological characteristics numerical and their examination gives important information about the effect of body structure on performance $(5,9)$.

Based on all of these information; in this study were aimed to determine that the relationship between anthropometric parameters with speed, which is a bio motor skill.

\section{MATERIAL \& METHOD}

One hundred-eighteen sedentary university students who are their gender 60 female and 58 male, their age $21.2 \pm 2.1$ year, their body weight $61.2 \pm 10.7 \mathrm{~kg}$, and their height $168.2 \pm 8.1 \mathrm{~cm}$ participated in this study voluntarily. Anthropometric measurements (circumference and length) and $30 \mathrm{~m}$ sprint test was performed in the research group.

Height of the research group was measured in $\mathrm{cm}$ by a nonflexible measuring tape of $0.1 \mathrm{~cm}$ sensitivity barefoot and by enabling anatomic posture, and their weight was measured in $\mathrm{kg}$ wearing shorts and $t$-shirt by an electronic scale of 100 gr sensitivity.

\section{Anthropometric}

Measurements:

Anthropometric measurements were made based on techniques suggested by International Biological Program (IBP)(Harvey, 1974) and "International Society for the Advancement of Kinanthropometry (ISAK)(23).

Stature (shoulder, chest, trunk, waist, hip, thigh, knee, calf and ankle) and length measurements (height, overarm, upper arm, fore arm, all arms, trunk, thigh, leg and whole leg) was made by an nonflexible measurement tape of $0.1 \mathrm{~cm}$ sensitivity.

All of the research group was performed a lumbering up protocol that included jogging 10 minutes and stretching exercise 10 minutes before 30 meter sprint test.

30 meter sprint test: Each volunteer in the research group ran in a tiled floor sports saloon in an area determined as $30 \mathrm{~m}$ with starting stand and maximum rigor. Running period was recorded in seconds by a Casio brand digital chronometer. This test was repeated three times by each volunteer and the best repeat test result was recorded.

Statistical analysis was made by SPSS 22.0 for Windows package program. Arithmetic mean, standard deviation, the lowest and highest values were determined. The relationship between anthropometric parameters and speed performance was tested by "Pearson Correlation". Margin of error $(\alpha)$ was regarded as $\mathrm{p}<0.05$ and $\mathrm{p}<0.01$.

\section{RESULTS}

Physical characteristics and anthropometric measurement values of the research group and other findings in the study are presented in the following tables.

Table 1. Physical characteristics of the women in research group.

\begin{tabular}{lccccc}
\hline Parameters & $\mathrm{n}$ & Minimum & Maximum & Mean & SD \\
Age (year) & 60 & 18.0 & 28.0 & 21.1 & 2.0 \\
Height $(\mathrm{cm})$ & 60 & 148.0 & 173.0 & 162.3 & 5.5 \\
$\begin{array}{l}\text { Body Weight } \\
(\mathrm{kg})\end{array}$ & 60 & 41.50 & 75.8 & 55.6 & 7.1 \\
\hline
\end{tabular}

Table 2. Circumference measurement values of the women in research group.

\begin{tabular}{lccccc}
\hline Parameters $(\mathrm{cm})$ & $\mathrm{n}$ & Minimum & Maximum & Mean & SD \\
\hline Shoulder & 60 & 83.0 & 102 & 93.5 & 4.3 \\
Chest & 60 & 75.0 & 90.0 & 82.9 & 4.1 \\
Trunk & 60 & 57.0 & 89.0 & 66.0 & 5.1 \\
Girth & 60 & 58.0 & 87.0 & 72.9 & 6.8 \\
Hip & 60 & 79.0 & 104.0 & 90.5 & 5.4 \\
Thigh & 60 & 41.0 & 96.0 & 49.9 & 7.3 \\
Knee & 60 & 29.0 & 38.0 & 33.8 & 2.1 \\
Calf & 60 & 20.0 & 39.0 & 32.9 & 3.1 \\
Ankle & 60 & 18.5 & 24.0 & 21.1 & 1.4 \\
\hline
\end{tabular}

Table 3. Length measurement values of the women in research group.

\begin{tabular}{lccccc}
\hline Parameters $(\mathrm{cm})$ & $\mathrm{n}$ & Minimum & Maximum & Mean & $\mathrm{SD}$ \\
\hline Overarm & 60 & 73.0 & 177.5 & 162.4 & 13.5 \\
Upper Arm & 60 & 30.0 & 40.0 & 36.3 & 1.9 \\
Fore Arm & 60 & 33.5 & 47.0 & 42.9 & 2.1 \\
Whole Arm & 60 & 70.5 & 86.0 & 79.1 & 3.4 \\
Trunk & 60 & 38.5 & 50.0 & 42.3 & 2.4 \\
Thigh & 60 & 36.0 & 49.5 & 40.9 & 2.7 \\
Calf & 60 & 31.0 & 45.0 & 37.8 & 2.8 \\
Whole Leg & 60 & 69.0 & 91.0 & 78.4 & 4.7 \\
\hline
\end{tabular}

Table 4. Sprint test values of the women in research group.

\begin{tabular}{llllll}
\hline Parameters (sec) & $\mathrm{n}$ & Minimum & Maximum & Mean & SD \\
\hline Speed Period & 118 & 4.91 & 6.31 & 5.55 & 0.30 \\
\hline
\end{tabular}


Table 5. Relationship between speed values and circumference and length measurements on women.

\begin{tabular}{|c|c|c|c|c|c|}
\hline Variables & & $\begin{array}{l}\text { Speed } \\
\text { Period }\end{array}$ & Variables & & $\begin{array}{l}\text { Speed } \\
\text { Period }\end{array}$ \\
\hline \multirow[t]{3}{*}{ Body Weight } & $\mathrm{r}$ & $.304^{*}$ & Height & $\mathrm{r}$ & .149 \\
\hline & $\mathrm{p}$ & .018 & & $\mathrm{p}$ & .255 \\
\hline & $\mathrm{n}$ & 118 & & $\mathrm{n}$ & 118 \\
\hline Shoulder & $\mathrm{r}$ & .190 & Overarm & $\mathrm{r}$ & -.004 \\
\hline \multirow[t]{2}{*}{ Circumference } & $\mathrm{p}$ & .146 & Length & $\mathrm{p}$ & .975 \\
\hline & $\mathrm{n}$ & 118 & & $\mathrm{n}$ & 118 \\
\hline Chest & $\mathrm{r}$ & .152 & Upper Arm & $\mathrm{r}$ & .082 \\
\hline \multirow[t]{2}{*}{ Circumference } & $\mathrm{p}$ & .248 & Length & $\mathrm{p}$ & .536 \\
\hline & $\mathrm{n}$ & 118 & & $\mathrm{n}$ & 118 \\
\hline Trunk & $\mathrm{r}$ & $.348^{*}$ & Forearm & $\mathrm{r}$ & .127 \\
\hline \multirow[t]{2}{*}{ Circumference } & $\mathrm{p}$ & .010 & Length & $\mathrm{p}$ & .332 \\
\hline & $\mathrm{n}$ & 118 & & $\mathrm{n}$ & 118 \\
\hline Girth & $\mathrm{r}$ & $.363^{* * *}$ & Whole Arm & $\mathrm{r}$ & $.297^{*}$ \\
\hline \multirow[t]{2}{*}{ Circumference } & $\mathrm{p}$ & .004 & Length & $\mathrm{p}$ & .021 \\
\hline & $\mathrm{n}$ & 118 & & $\mathrm{n}$ & 118 \\
\hline Hip & $\mathrm{r}$ & $.369^{* *}$ & Trunk & $\mathrm{r}$ & $.263^{*}$ \\
\hline \multirow[t]{2}{*}{ Circumference } & $\mathrm{p}$ & .004 & Length & $\mathrm{p}$ & .042 \\
\hline & $\mathrm{n}$ & 118 & & $\mathrm{n}$ & 118 \\
\hline Thigh & $\mathrm{r}$ & .020 & Thigh & $\mathrm{r}$ & -.018 \\
\hline \multirow[t]{2}{*}{ Circumference } & $\mathrm{p}$ & .879 & Length & $\mathrm{p}$ & .094 \\
\hline & $\mathrm{n}$ & 118 & & $\mathrm{n}$ & 118 \\
\hline \multirow{3}{*}{$\begin{array}{l}\text { Knee } \\
\text { Circumference }\end{array}$} & $\mathrm{r}$ & $.270^{*}$ & Calf Length & $\mathrm{r}$ & .218 \\
\hline & $\mathrm{p}$ & .037 & & $\mathrm{p}$ & .095 \\
\hline & $\mathrm{n}$ & 118 & & $\mathrm{n}$ & 118 \\
\hline Calf & $\mathrm{r}$ & $.293^{*}$ & Whole Leg & $\mathrm{r}$ & .071 \\
\hline \multirow[t]{2}{*}{ Circumference } & $\mathrm{p}$ & .023 & Length & $\mathrm{p}$ & .591 \\
\hline & $\mathrm{n}$ & 118 & & n & 118 \\
\hline \multirow{3}{*}{$\begin{array}{l}\text { Ankle } \\
\text { Circumference }\end{array}$} & $\mathrm{r}$ & $.282^{*}$ & & & \\
\hline & $\mathrm{p}$ & .029 & & & \\
\hline & $\mathrm{n}$ & 118 & & & \\
\hline
\end{tabular}

${ }^{*} \mathrm{p}<0.05 ;{ }^{* *} \mathrm{p}<0.01$

Table 6. Physical characteristics of the men in research group.

\begin{tabular}{lccccc}
\hline Parameters & $\mathrm{n}$ & Minimum & Maximum & Mean & SD \\
\hline Age (year) & 58 & 18.0 & 30.0 & 21.2 & 2.1 \\
Height $(\mathrm{cm})$ & 58 & 158.5 & 190.0 & 174.3 & 5.4 \\
Body Weight $(\mathrm{kg})$ & 58 & 51.4 & 92.90 & 68.9 & 9.7 \\
\hline
\end{tabular}

Table 7. Circumference measurement values of the men in research group.

\begin{tabular}{lccccc}
\hline $\begin{array}{l}\text { Parameters } \\
(\mathrm{cm})\end{array}$ & $\mathrm{n}$ & Minimum & Maximum & Mean & SD \\
\hline Shoulder & 58 & 92.0 & 130.0 & 108.5 & 6.5 \\
Chest & 58 & 76 & 107.0 & 88.4 & 6.2 \\
Trunk & 58 & 63.0 & 98.0 & 75.7 & 6.5 \\
Girth & 58 & 64.0 & 99.0 & 79.6 & 7.0 \\
Hip & 58 & 79.0 & 105.0 & 91.5 & 5.4 \\
Thigh & 58 & 40.0 & 83.0 & 50.9 & 6.2 \\
Knee & 58 & 30.5 & 41.0 & 35.2 & 2.0 \\
Calf & 58 & 25.0 & 43.0 & 34.9 & 3.1 \\
Ankle & 58 & 17.5 & 31.0 & 22.6 & 2.1 \\
\hline
\end{tabular}

On women, it was established that there was a medium positive relationship between trunk circumference, girth circumference, hip circumference values and sprint period values (0.01). Besides, there was a weak positive significant relationship between knee circumference, calf circumference, ankle circumference, whole arm length, trunk length values and sprint period values (0.05).

On men, it was found that there were a no significant relationship anthropometric parameter values and sprint period values (0.05).

Without gender gap, according to statistical analysis findings; it was determined that there was a strong negative relationship shoulder circumference, chest circumference, height, forearm length, trunk length values and sprint period values (0.01) besides there was a medium negative significant relationship body weight, chest circumference, whole arm length, whole leg length values and sprint period values (0.05). Moreover, it was found that there was a weak negative significant relationship girth circumference, calf length, overarm length values and sprint period values (0.01); in addition, there was a weak significant relationship calf circumference, thigh circumference values and sprint period values (0.05).

Table 8. Length measurement values of the men in research group.

\begin{tabular}{lccccc}
$\begin{array}{l}\text { Parameters } \\
(\mathrm{cm})\end{array}$ & $\mathrm{n}$ & Minimum & Maximum & Mean & SD \\
\hline Overarm & 58 & 82.0 & 192.5 & 174.9 & 18.6 \\
Upper Arm & 58 & 32.0 & 44.0 & 38.4 & 2.3 \\
Fore Arm & 58 & 40.0 & 52.5 & 47.3 & 2.2 \\
Whole Arm & 58 & 75.0 & 95.5 & 85.5 & 4.2 \\
Trunk & 58 & 39.5 & 53.0 & 47.1 & 3.1 \\
Thigh & 58 & 38.0 & 50.0 & 42.5 & 2.8 \\
Calf & 58 & 35.0 & 46.5 & 40.3 & 2.6 \\
Whole Leg & 58 & 71.0 & 91.0 & 82.5 & 4.2 \\
\hline \multicolumn{7}{c}{} & & & & \\
Table 9. Sprint test values of the men in research group. \\
\hline Parameters (sec) & $\mathrm{n}$ & Minimum & Maximum & Mean & SD \\
\hline Speed Period & 58 & 4.10 & 5.56 & 4.51 & 0.25 \\
\hline
\end{tabular}


Table 10. Relationship between speed values and circumference and length measurements on men.

\begin{tabular}{|c|c|c|c|c|c|}
\hline Variables & & $\begin{array}{l}\text { Speed } \\
\text { Period }\end{array}$ & Variables & \multicolumn{2}{|r|}{$\begin{array}{l}\text { Speed } \\
\text { Period }\end{array}$} \\
\hline \multirow[t]{3}{*}{ Body Weight } & $\mathrm{r}$ & .213 & Height & $\mathrm{r}$ & .049 \\
\hline & $\mathrm{p}$ & .109 & & $\mathrm{p}$ & .715 \\
\hline & $\mathrm{n}$ & 58 & & $\mathrm{n}$ & 58 \\
\hline Shoulder & $\mathrm{r}$ & .101 & Overarm & $\mathrm{r}$ & .217 \\
\hline \multirow[t]{2}{*}{ Circumference } & $\mathrm{p}$ & .451 & Length & $\mathrm{p}$ & .101 \\
\hline & n & 58 & & n & 58 \\
\hline Chest & $\mathrm{r}$ & .019 & Upper Arm & $\mathrm{r}$ & .077 \\
\hline \multirow[t]{2}{*}{ Circumference } & $\mathrm{p}$ & .888 & Length & $\mathrm{p}$ & .567 \\
\hline & n & 58 & & n & 58 \\
\hline Trunk & $\mathrm{r}$ & .005 & Forearm & $\mathrm{r}$ & .051 \\
\hline \multirow[t]{2}{*}{ Circumference } & $\mathrm{p}$ & .973 & Length & $\mathrm{p}$ & .702 \\
\hline & $\mathrm{n}$ & 58 & & $\mathrm{n}$ & 60 \\
\hline Girth & $\mathrm{r}$ & .122 & Whole Arm & $\mathrm{r}$ & -.016 \\
\hline \multirow[t]{2}{*}{ Circumference } & $\mathrm{p}$ & .362 & Length & $\mathrm{p}$ & .904 \\
\hline & n & 58 & & n & 58 \\
\hline Hip & $\mathrm{r}$ & .174 & Trunk & $\mathrm{r}$ & .088 \\
\hline \multirow[t]{2}{*}{ Circumference } & $\mathrm{p}$ & .192 & Length & $\mathrm{p}$ & .509 \\
\hline & $\mathrm{n}$ & 58 & & $\mathrm{n}$ & 58 \\
\hline Thigh & $\mathrm{r}$ & -.046 & Thigh & $\mathrm{r}$ & .061 \\
\hline \multirow[t]{2}{*}{ Circumference } & $\mathrm{p}$ & .731 & Length & $\mathrm{p}$ & .651 \\
\hline & n & 58 & & $\mathrm{n}$ & 58 \\
\hline Knee & $\mathrm{r}$ & .165 & Calf Length & $\mathrm{r}$ & .163 \\
\hline \multirow[t]{2}{*}{ Circumference } & $\mathrm{p}$ & .214 & & $\mathrm{p}$ & .221 \\
\hline & $\mathrm{n}$ & 58 & & $\mathrm{n}$ & 58 \\
\hline Calf & $\mathrm{r}$ & .031 & Whole Leg & $\mathrm{r}$ & .216 \\
\hline \multirow[t]{2}{*}{ Circumference } & $\mathrm{p}$ & .815 & Length & $\mathrm{p}$ & .104 \\
\hline & $\mathrm{n}$ & 58 & & $\mathrm{n}$ & 58 \\
\hline Ankle & $\mathrm{r}$ & .026 & & & \\
\hline \multirow[t]{2}{*}{ Circumference } & $\mathrm{p}$ & .849 & & & \\
\hline & $\mathrm{n}$ & 58 & & & \\
\hline
\end{tabular}

Table 11. Physical characteristics of the research group.

\begin{tabular}{lccccc}
\multicolumn{6}{l}{ Table 11. Physical characteristics of the research group. } \\
\hline Parameters & $\mathrm{n}$ & Minimum & Maximum & Mean & SD \\
\hline Age (year) & 118 & 18.0 & 30.0 & 21.2 & 2.1 \\
Height $(\mathrm{cm})$ & 118 & 148.0 & 190.0 & 168.2 & 8.1 \\
$\begin{array}{l}\text { Body Weight } \\
\text { (kg) }\end{array}$ & 118 & 41.50 & 92.90 & 61.2 & 10.7 \\
\hline
\end{tabular}

Table 12. Circumference Measurement Values of the Research Group

\begin{tabular}{lccccc}
\hline $\begin{array}{l}\text { Parameters } \\
(\mathrm{cm})\end{array}$ & $\mathrm{n}$ & Minimum & Maximum & Mean & SD \\
\hline Shoulder & 118 & 83.0 & 130.0 & 100.9 & 9.3 \\
Chest & 118 & 75.0 & 107.0 & 85.63 & 6.0 \\
Trunk & 118 & 57.0 & 98.0 & 70.8 & 7.7 \\
Girth & 118 & 58.0 & 99.0 & 76.2 & 7.6 \\
Hip & 118 & 79.0 & 105.0 & 91.0 & 5.4 \\
Thigh & 118 & 40.0 & 96.0 & 50.4 & 6.8 \\
Knee & 118 & 29.0 & 41.0 & 34.5 & 2.2 \\
Calf & 118 & 20.0 & 43.0 & 33.9 & 3.3 \\
Ankle & 118 & 17.5 & 31.0 & 21.8 & 1.9 \\
\hline
\end{tabular}

Table 13. Length measurement values of the research group.

\begin{tabular}{lccccc}
\hline $\begin{array}{l}\text { Parameters } \\
(\mathrm{cm})\end{array}$ & $\mathrm{n}$ & Minimum & Maximum & Mean & SD \\
\hline Overarm & 118 & 73.0 & 192.5 & 168.6 & 17.3 \\
Upper Arm & 118 & 30.0 & 66.0 & 37.6 & 3.5 \\
Fore Arm & 118 & 33.5 & 52.5 & 45.1 & 3.1 \\
Whole Arm & 118 & 46.0 & 95.5 & 82.1 & 6.1 \\
Trunk & 118 & 38.5 & 53.0 & 44.6 & 3.7 \\
Thigh & 118 & 36.0 & 50.0 & 41.7 & 2.8 \\
Calf & 118 & 31.0 & 46.5 & 39.0 & 3.0 \\
Whole Leg & 118 & 69.0 & 91.0 & 80.4 & 4.9 \\
\hline
\end{tabular}

Table 14. Sprint test values of the research group.

\begin{tabular}{lccccc}
\hline Parameters $(\mathrm{sec})$ & $\mathrm{n}$ & Minimum & Maximum & Mean & $\mathrm{SD}$ \\
\hline Speed Period & 118 & 4.10 & 6.31 & 5.04 & 0.60 \\
\hline
\end{tabular}

Table 15. Relationship between research groups' speed values and circumference and length measurements.

\begin{tabular}{|c|c|c|c|c|c|}
\hline Variables & & $\begin{array}{l}\text { Speed } \\
\text { Period }\end{array}$ & Variables & & $\begin{array}{l}\text { Speed } \\
\text { Period }\end{array}$ \\
\hline \multirow[t]{3}{*}{ Body Weight } & $\mathrm{r}$ & $-.451^{* *}$ & \multirow[t]{3}{*}{ Height } & $\mathrm{r}$ & $-.618^{* *}$ \\
\hline & $\mathrm{p}$ & .000 & & $\mathrm{p}$ & .000 \\
\hline & $\mathrm{n}$ & 60 & & $\mathrm{n}$ & 60 \\
\hline Shoulder & $\mathrm{r}$ & $-.672^{* *}$ & Overarm & $\mathrm{r}$ & $-.269^{* *}$ \\
\hline \multirow[t]{2}{*}{ Circumference } & $\mathrm{p}$ & .000 & \multirow[t]{2}{*}{ Length } & $\mathrm{p}$ & .003 \\
\hline & $\mathrm{n}$ & 60 & & $\mathrm{n}$ & 60 \\
\hline Chest & $\mathrm{r}$ & $-.383^{* *}$ & Upper Arm & $\mathrm{r}$ & -.156 \\
\hline \multirow[t]{2}{*}{ Circumference } & $\mathrm{p}$ & .000 & \multirow[t]{2}{*}{ Length } & $\mathrm{p}$ & .091 \\
\hline & $\mathrm{n}$ & 60 & & $\mathrm{n}$ & 60 \\
\hline Trunk & $\mathrm{r}$ & $-.507^{* *}$ & Forearm & $\mathrm{r}$ & $-.587^{* *}$ \\
\hline \multirow[t]{2}{*}{ Circumference } & $\mathrm{p}$ & .000 & \multirow[t]{2}{*}{ Length } & $\mathrm{p}$ & .000 \\
\hline & $\mathrm{n}$ & 60 & & $\mathrm{n}$ & 60 \\
\hline Girth & $\mathrm{r}$ & $-.282^{* *}$ & Whole Arm & $\mathrm{r}$ & $-.446^{* *}$ \\
\hline \multirow[t]{2}{*}{ Circumference } & $\mathrm{p}$ & .002 & \multirow[t]{2}{*}{ Length } & $\mathrm{p}$ & .000 \\
\hline & $\mathrm{n}$ & 60 & & $\mathrm{n}$ & 60 \\
\hline Hip & $\mathrm{r}$ & .049 & \multirow[t]{3}{*}{ Trunk Length } & $\mathrm{r}$ & $-.519^{* *}$ \\
\hline \multirow{2}{*}{ Circumference } & $\mathrm{p}$ & .596 & & $\mathrm{p}$ & .000 \\
\hline & $\mathrm{n}$ & 60 & & $\mathrm{n}$ & 60 \\
\hline Thigh & $\mathrm{r}$ & -.066 & \multirow[t]{3}{*}{ Thigh Length } & $\mathrm{r}$ & $-.230^{*}$ \\
\hline \multirow[t]{2}{*}{ Circumference } & $\mathrm{p}$ & .477 & & $\mathrm{p}$ & .012 \\
\hline & $\mathrm{n}$ & 60 & & $\mathrm{n}$ & 60 \\
\hline \multirow{3}{*}{$\begin{array}{l}\text { Knee } \\
\text { Circumference }\end{array}$} & $\mathrm{r}$ & -.176 & \multirow[t]{3}{*}{ Calf Length } & $\mathrm{r}$ & $-.290^{* *}$ \\
\hline & $\mathrm{p}$ & .057 & & $\mathrm{p}$ & .001 \\
\hline & $\mathrm{n}$ & 60 & & $\mathrm{n}$ & 60 \\
\hline Calf & $\mathrm{r}$ & $-.193^{*}$ & Whole Leg & $\mathrm{r}$ & $-.310^{* *}$ \\
\hline \multirow[t]{2}{*}{ Circumference } & $\mathrm{p}$ & .036 & \multirow[t]{5}{*}{ Length } & $\mathrm{p}$ & .001 \\
\hline & $\mathrm{n}$ & 60 & & $\mathrm{n}$ & 60 \\
\hline Ankle & $\mathrm{r}$ & -.162 & & & \\
\hline \multirow[t]{2}{*}{ Circumference } & $\mathrm{p}$ & .080 & & & \\
\hline & $\mathrm{n}$ & 60 & & & \\
\hline
\end{tabular}

${ }^{*} \mathrm{p}<0.05 .{ }^{* *} \mathrm{p}<0.01$

\section{DISCUSSION}

A study conducted on Physical education and sport high School students by Bozlar has found that the general age average of students was $20.52 \pm 0.55$ year. The average weight of students was 
$71.028 \pm 1.84 \mathrm{~kg}$, and the average height of students was $174.38 \pm 1.39 \mathrm{~cm} \mathrm{(6).}$

A similar study conducted on university students by Yaprak has found that the age average of students was $22.84 \pm 2.42$ year, the average weight of students was $62.27 \pm 880 \mathrm{~kg}$, and the average height of students was $169.42 \pm 7.21 \mathrm{~cm}$ (34).

The another similar study conducted on university students by Bostanc1 et al. has found that the general age average of students was $22.27 \pm 2.20$ year. The average weight of students was $66.77 \pm$ $8.12 \mathrm{~kg}$, and the average height of students was $172.36 \pm 6.14 \mathrm{~cm} \mathrm{(4)}$.

A different study conducted on university students by Yorulmaz et al. has found that the general age average of students was $19.84 \pm 1.33$ year.The average weight of students was $68.42 \pm 8.23$ $\mathrm{kg}$, and the average height of students was $170.38 \pm 8.19 \mathrm{~cm}$ (35).

The another different study conducted on KTÜ Giresun Education Faculty students by Kalkavan et al. has found that the general age average of students was 22.2year, the average weight of students was $67.8 \mathrm{~kg}$, and the average height of students was $1.67 \mathrm{~m}$ (13).

The end of this study, physical education and sport high School students has found that the general age average of students was $21.2 \pm 2.1$ year. The average weight of students was $61.2 \pm 10.7 \mathrm{~kg}$, and the average height of students was $168.2 \pm 8.1$ $\mathrm{cm}$. In addition to that Female physical education and sport high School students has found that the general age average of students was $21.1 \pm 2.0$ year. The average body weight of students was $55.6 \pm 7.1$ $\mathrm{kg}$. and the average height of students was $162.3 \pm$ $5.5 \mathrm{~cm}$. and male physical education and sport high School students has found that the general age average of students was $21.2 \pm 2.1$ year. The average weight of students was $68.9 \pm 9.7 \mathrm{~kg}$, and the average height of students was $174.3 \pm 5.4 \mathrm{~cm}$. The results are parallel to others study in related body of literature.

Number of studies scrutinizing the relationship between anthropometric characteristics and speed performance is low considerably. Therefore, the discussion was made directly only along with the studies investigating the relationship between anthropometric characteristics and speed performance indirectly.
Physical structure is only one of the factors affecting performance and success. Physical structure combines with strength, power, flexibility, speed, resistance and swiftness, which are among motor characteristics necessary for the sports branch, and influences the success of the athlete positively(30).

In addition to such factors for success in sports performance or sports, anthropometric characteristics also have a role. In general, when these attributes which are determined genetically are evaluated well, they are provided for performance in a certain sports branch(33).

It is known that each sport has unique characteristics. It is also known that these attributes of athletes with special physical structure appropriate to the branch are effective for success. Knowing physical characteristics of successful athletes in skill selection could be a model. For this reason, it is important to take measurements of length. Circumference, etc., among physical attributes $(7,27)$.

Especially performance and strength generation is directly related to height, body weight, arm and leg lengths, joint mobility, and flexibility levels(1).

Determination of anthropometric profile facilitates determination of training process and skills of the players. When anthropometric body measurements are taken as the basis by using standard methods, athletes display high degree performance(21).

Several studies have assessed the anthropometric, physiological, psychological, and motor skill attributes of individual sports $(3,9,15,18,25,31)$.

The statistical analysis results of this study have corroborated in the other studies in related body of literature.

In conclusion; it was found that there was a significant relationship between the speed values of the study group and body weight, shoulder, chest, trunk, waist circumference $(\mathrm{p}<0.01)$ and calf circumference $(p<0.05)$. And there was a significant relationship between the speed values of the study group and height, fathom length, forearm length, whole arm length, trunk length, calf length $(p<0.01)$ and thigh length $(\mathrm{p}<0.05)$. Based on these results; it can be stated that when circumference and length measurements increase, speed performance could increase as well. 


\section{REFERENCES}

1. Astrand PO, Roah K 1986. Textbook of Work Physiology. Third edition. Mc.Graw Hill Company New York. p.18-141.

2. Behnke. AR, Royce J. Body size, shape and composition of several types of athletes. J. Sports. Med. 1966; 6:75.

3. Bencke J, Damsgaard R, Saekmose A, Jorgensen P, Jorgesen $\mathrm{K}$, Klausen K. Anaerobic power and muscle strength characteristics of 11 year old elite and non-elite boys and girls from gymnastics, team handball, tennis and swimming. Scandinavian Journal of Medicine and Science in Sports, 2002; 12: $171-178$.

4. Bostancı Ö, Uzun A, Emirzeoğlu M, Kabadayı M, Şahin B, Bilgiç $\mathrm{S}$. To examine the height-body relations of footballers who are students at physical education and sports with some anthropom. Journal of Physical Education and Sport Sciences, 2004; 6(2): 26-34.

5. Bourgois J, Claessens AL, Vrijens J, Philippaerts R, Renterghem BV, Thomis M, Janssens M, Loos R, Lefevre J. Anthropometric characteristics of elite male junior rowers. British Joournal of Sports Medicine, 2002; 34(3):213-217.

6. Bozlar O. Investigation of the physical education and sports high school students their anthropometric features and Somatotype characteristics. Master Thesis. Karadeniz Technical University. Education Science Institute. Department of Physical Education and Sports Teacher. Trabzon. 2011.

7. Claessens AL. Talent detection and talent development: kinanthropometric issues. Acta Kinesiologiae Universitatis Tartuensis. 1999; 4: 47-64.

8. Ergen E, Sardella F, Monte AD. The relationship of maxima alactacid anaerobic power to somatotype in trained subjects. Br. J. Sports Med, 1985; 19(4): 221.

9. Gabbett TJ. Physiological and anthropometric characteristics of amateur rugby league players. Br J Sports Med, 2000; 34(4):303-307.

10. Harvey RG. An anthropometric survey of growth and physique of the populations of KarKar Island and Lufa Subdistrict. New Guinea. Phil. Trans. R. Soc. B 1974; 268: 279292.

11. Heimer S, Misigoj M, Medved V. Some anthropological of top volleyball players in SFR Yugoslavia. The Journal of Sports Medicine Fitness,1988; 28: 200-208.

12. Hopper MN. Somatotype in high performance female netball players. BJSP Med, 1977; 31: 197-199.

13. Kalkavan A, Yaman M, Karakuş S. Torun CK. Yaman Ç. CihanH. Investigation of the KTU Giresun Education Faculty students their anthropometric features and Somatotype characteristics. Journal of Gazi University Physical Education and Sports Science, 1977; 2(1): 1-8.

14. Kidd D, Winter M. Some anthropometric characteristics of the national junior hammer squad. Br J Sports Med, 1983; 17: 152.

15. Kukolj M, Ugarkovic D, Jaric S. Profiling anthropometric characteristics and functional performance of 12 to 18-yearold elite junior soccer players. Journal of Human Movement Studies, 2003; 45:403-418.

16. Malina RM. Anthropometric correlates of strength and motor performance. Exercise and Sports Science Review. 1975; 3 249.
17. Malina RM. Maturatural Considerations in Elite Young Athletes. In Perspectives in Kinanthropometry (edited by J. A. P. Day). Champaign. III: Human Kinetics,1986; 29-43.

18. Mohamed H, Vaeyens R, Matthys S, Multael M, Lefevre J, Lenoir M., Philippaerts R. Anthropometric and performance measures for the development of a talent detection and identification model in youth handball. Journal of Sports Sciences, 2009;27(3): 257-266.

19. Ozer K. Kinanthropometry. Morphological Planning in Sports. Nobel Publishing Distribution Trade Ltd. Co.,2009, pp. 2-6.

20. Parizkova J. Body composition and physical fitness. Curr Anthrop, 1968; 9:273.

21. Poliszczuk T, Mosakowska M. Anthropometric profile of Polish elite badminton players. Medsportpress,2010; 1(6): 4546.

22. Ross WD, Marfell-Jones MJ. 1991. Kinanthropometry. Physiological Testing of the High-Performance Athlete. Ed. MacDougall. J.D.. Wenger. HA,Gren HJ, 2. Edith. Illinois. Human Kinetics Books, 1991

23. Ross WD, Marfell-Jonsen MJ. 1991. Kinanthropometry. Physiological Testing of the High-Performance Athlete. Editor: MacDougall. JD, Wenger HA, Green. H.J. Human kinetics Books. Champain, Illnois.

24. Ross WO. Brown SR, Yu JW. Faulkner RA. 1977. Somatotype of Canadian figure skaters. J Sports Med, 17: 195.

25. Saenz-Lopez P, Ibanez SJ, Gimenez J, Sierra A, Sanchez M. Multifactor characteristics in the process of development of the male expert basketball player in Spain. International Journal of Sport Psychology, 2005; 36. 151-171.

26. Sharma SS, Dixit NK. Somatotype of athletes and their performance. Int. J. Sports Med. 1985; 6 (3): 16.

27. Slater GJ, Rice AJ, Mujika, I, Hahn AG, Sharpe K, Jenkins, DG. Physique traits of lightweight rowers and their relationship to competitive success. British Journal of Sports Medicine, 2005; 39(10):736-4.

28. Slaughter MH, Lahmann TC, Misner JE. Association of somatotype and body composition to physical performance in 7-12 year-old girls. J Sports Med, 1980; 20: 189.

29. Sodhi HS. Physique of top-ranking Indian wrestlers. J Sports Med, 1983; 23:59.

30. Song Thomas MK, Cipriano N. Effects of seasonal training on physical and physiological anaerobic performance of elite young wrestlers. Journal of Sports and Medicine, 2007; 6(Css12): $34-38$

31. Thelwell RC, Greenlees IA, Weston NJV. Using psychological skills training to develop soccer performance. Journal of Applied Sport Psychology, 2006; 18: 254-270.

32. Toriola AL, Salokun SO, Mathur DN. Somatotype characteristics of male sprinters, basketball, soccer and field hockey players. Int J Sports Med, 1985; 6(6): 344

33. Weineck J. Functional Anatomy in Sports. Translator: Elmaci AS, Ankara: Bagirgan Publishing House, 1998.

34. Yaprak Y, Aslan A, Taşer H. Considering the relationship between $\mathrm{VO} 2 \max$, cardiac output, pulmonary function and anthropometric measurements at university students. Journal of Physical Education and Sport Sciences, 2008; 10(1): 12-20. 
35. Yorulmaz F, Taşkınalp O, Yaprak M, Turut M, Mesut R. Some anthropometric features of male medical school students from Trakya. Balkan Medical Journal, 1998; 3: 85-90. 